\title{
GAGUEIRA E DISFLUÊNCIA COMUM NA INFÂNCIA: ANÁLISE DAS MANIFESTAÇÕES CLÍNICAS NOS SEUS ASPECTOS QUALITATIVOS E QUANTITATIVOS
}

\author{
Stuttering and Common Dysfluency in Childhood: analyses of \\ clinical manifestations in their qualitative and quantitative aspects
}

\author{
Suzana Maria de Amarante Merçon ${ }^{(1)}$, Katia Nemr ${ }^{(2)}$
}

\begin{abstract}
RESUMO
Objetivo: analisar em seus aspectos qualitativos e quantitativos as manifestações clínicas da gagueira e da disfluência comum na faixa etária de dois a seis anos. Métodos: revisão de Literatura Sistemática a partir de livros e artigos científicos de Fonoaudiologia indexados no LILACS e no MEDLINE de 1993 a 2005. Resultados: as diferenças qualitativas mais importantes são: o tipo de unidade lingüística na qual as disfluências ocorrem, tipologias de disfluências, presença ou ausência de esforço físico durante a fala e possíveis dificuldades na linguagem. Pré-escolares com gagueira freqüentemente apresentam dificuldades em competências metalinguísticas, especialmente com as metafonológicas, sendo que mais estudos sobre este aspecto são necessários. A freqüência de sílabas disfluentes e a taxa de elocução verbal estão entre os parâmetros quantitativos significativos. Conclusão: diferenças na fala e na linguagem parecem ser fatores importantes para distinguir gagueira de disfluências comuns infantis.
\end{abstract}

DESCRITORES: Gagueira; Pré-Escolar; Linguagem Infantil; Fonética; Genética

\section{INTRODUÇÃO}

Os profissionais que lidam com crianças disfluentes questionam-se com reiteração em como distinguir as manifestações clínicas próprias da gagueira daquelas que são comuns e que geralmente são detectadas no decorrer do desenvolvimento da fala e da linguagem. Alguns autores argumentam que não é tão simples este diagnóstico diferencial, mas que é muito importante que seja feito precocemente já que o quadro de gagueira algumas vezes se agrava e cronifica levando a criança a enfrentar diversas dificuldades nas suas relações de comunicação ${ }^{1-5}$.

Observou-se que a quebra da fluência é algo habitual na fala das crianças em idade de estruturação da linguagem, o que contribui para confirmar o termo

(1) Fonoaudióloga do Colégio Nossa Senhora de Lourdes - Rio de Janeiro; Graduada em Medicina pela Universidade Federal Fluminense; Especializanda em Linguagem.

(2) Fonoaudióloga Professora Concursada da Universidade de São Paulo; Doutora em Psicologia Social pela Universidade de São Paulo. "disfluência comum", e que difere em vários aspectos quando comparadas com aquelas consideradas gagas. Quais seriam estas diferenças? Um substancial volume de evidências clínicas fornece a base para que se realize $o$ atendimento especializado precoce da gagueira, que se inicia geralmente entre os dois e cinco anos de idade, principalmente por volta dos três anos. Estas evidências clínicas encaixam-se dentro de parâmetros qualitativos e quantitativos, havendo quase sempre influência de fatores orgânicos, como os genéticos, e também de sociais e emocionais para sua expressão ${ }^{1-12}$.

Processos de maturação fisiológicos e neuroanatômicos provavelmente estão relacionados com o surgimento da gagueira desenvolvimental em crianças pré-escolares, a qual parece estar intimamente vinculada às habilidades metalingüísticas. Estudos recentes de imagem cerebral indicam que a gagueira possivelmente tem sua origem nos múltiplos centros cerebrais de linguagem, ainda que se aceite que haja também dificuldades no controle motor da fala. Assim sendo, um meio ambiente acolhedor pode minimizar as manifestações de gagueira, mas 
não impedir que ela ocorra. Mesmo com essa perspectiva predominantemente neurolingüística, a influência dos outros fatores continua sendo aceita, assim, a idéia da multicausalidade para a gagueira de desenvolvimento permanece válida ${ }^{1,3}$.

Analisar as manifestações clínicas respeitando determinados parâmetros é fundamental uma vez que as condutas a serem tomadas dependerão da tipologia e da freqüência das disfluências e do modo como a criança reage às dificuldades na fala. E, caso a criança apresente apenas disfluências comuns na fala, o fonoaudiólogo deve estar preparado para orientar os responsáveis. Adultos podem propiciar relações de comunicação mais favoráveis para o desenvolvimento da habilidade da fluência com a redução de exigências exageradas perante o desempenho da criança no diálogo e focalizando a atenção no conteúdo de sua fala, não no modo como ela fala ${ }^{12,13}$.

O presente estudo tem como objetivo principal identificar e analisar qualitativamente e quantitativamente os aspectos clínicos que possibilitam realizar o diagnóstico diferencial entre a gagueira infantil e a disfluência comum na infância.

\section{MÉTODOS}

Trata-se de uma Revisão de Literatura Sistemática baseada no que foi publicado a respeito do assunto nos últimos doze anos, com análise descritiva. Foram pesquisados Livros e Artigos científicos (indexados) de Fonoaudiologia que abordam o tema e/ou fornecem subsídios para a elaboração deste trabalho. Os artigos científicos foram obtidos a partir de pesquisa nas bases de dados LILACS e MEDLINE no período de 1993-2005 com os seguintes descritores: Gagueira; Disfluência.

\section{RESULTADOS}

Várias pesquisas demonstraram que existem diferenças tanto no aspecto qualitativo como no quantitativo entre a gagueira infantil e a disfluência comum na infância. Para que a distinção precoce entre as duas condições seja possível, é preciso identificar as manifestações clínicas e então realizar uma análise comparativa entre elas ${ }^{2,4,5}$.

\section{Análise Comparativa Qualitativa}

Há diferenças qualitativas quanto à forma de apresentação e evolução clínica. Enquanto as manifestações clínicas da gagueira infantil estão mais presentes do que ausentes na fala da criança, embora com variações significativas na sua ocorrência de acordo com a sua intensidade ${ }^{5}$, as disfluências comuns na infância são, na maioria das vezes, esporádicas ${ }^{5,7,9}$. Quanto à evolução clínica, a gagueira infantil mais freqüentemente tem um desenvolvimento gradual, podendo também, mais raramente, surgir abruptamente ${ }^{1}$. Nos estágios iniciais podem ocorrer períodos de remissão parcial, conferindo à gagueira infantil um caráter intermitente ${ }^{3}$. Embora possa haver uma recuperação espontânea (na maior parte dos casos), quando isso não acontece, há a possibilidade de ocorrer o agravamento do quadro de gagueira ${ }^{4,5,7,12}$, enquanto na disfluência comum detecta-se, em geral, uma progressiva aquisição de maior fluência ${ }^{1,3-5,9,12}$.

Uma importante diferença qualitativa é o tipo de unidade lingüística na qual as disfluências ocorrem: fones e sílabas na gagueira infantil, enquanto na disfluência comum trata-se da palavra inteira, sintagma e/ ou sentença, principalmente ${ }^{1,3,4,10,14-16}$. Constatou-se numa pesquisa, que $97,8 \%$ dos eventos de gagueira ocorreram nas primeiras sílabas das palavras e $76,5 \%$ no primeiro fone das sílabas, demonstrando um claro "efeito de início de palavra" interferindo na ocorrência de gagueira em crianças em idade pré-escolar, o que não se observa na disfluência comum ${ }^{16}$.

As tipologias de disfluências mais freqüentemente observadas também diferem, em cada quadro. Os principais tipos de disfluências gagas são: repetições de fones ou de sílabas dentro de uma mesma palavra, repetições de partes de palavras e ainda, prolongamentos de fones e bloqueios. São freqüentes também as pausas preenchidas. $E$, os principais tipos de disfluências comuns são: repetições de palavras inteiras, de sentenças e/ou de sintagmas, revisões de palavras, palavras não terminadas, pausas silenciosas hesitativas e pausas preenchidas. Na gagueira infantil, as rupturas na fala são involuntárias, enquanto nas disfluências comuns, ainda que a criança apresente pouco controle sobre as disfluências, há um certo grau de deliberação ${ }^{1-5,7,10,12,14}$.

A criança pré-escolar com gagueira pode apresentar tensões faciais e/ou corporais. Movimentos corporais associados (chamados também de comportamentos acessórios ou secundários) podem surgir e se intensificar, com a evolução da gagueira. $\mathrm{Na}$ disfluência comum, não há evidência de tensões faciais e/ou corporais, como também não há comportamentos acessórios ${ }^{1-10,12,15,17}$.

Crianças pequenas com gagueira podem manifestar ainda: impaciências em momentos de fala, dificuldades em manter o contato visual no diálogo e no relacionamento com outras pessoas, podendo até desistir de falar ${ }^{7}$. Nesses casos com freqüência se observa incoordenação entre a fala e a respiração, enquanto na disfluência comum a respiração é regular e contínua, mesmo durante as repetições ${ }^{2,3,7,9,12,15}$.

A gagueira em pré-escolares difere da disfluência comum na infância também quanto a aspectos qualitativos lingüísticos. Pesquisas realizadas com crianças com gagueira revelaram que 
muitas delas apresentam comprometimento da linguagem o que não foi observado em crianças com disfluência comum ${ }^{3,17-21}$.

Crianças que gaguejam em idade pré-escolar demonstraram dificuldades no desempenho em tarefas metalingüísticas, o que não foi detectado, na mesma intensidade, em crianças sem gagueira, incluindo crianças com disfluências comuns, na mesma faixa etária. Essas pesquisas também revelaram que tais dificuldades encontravam-se nas construções gramaticais, quando as crianças com gagueira tiveram maiores dificuldades para avaliar frases irregulares nos aspectos semântico e sintático. Outro estudo, com crianças com idades entre 3;3 e 5;5 (anos; meses) mostrou que as dificuldades metalingüísticas em crianças com gagueira, quando comparadas com aquelas sem gagueira (estando na idade de disfluências comuns), foram maiores e mais especificamente notadas em tarefas envolvendo o planejamento e a recuperação, de forma rápida e correta, de unidades estruturais de frases, o que, segundo os autores, poderia estar prejudicando a fluência no discurso ${ }^{19,20}$.

Pesquisas com pré-escolares com gagueira com idade média de 5;10 (anos; meses) evidenciaram que estas têm dificuldades em habilidades metafonológicas também. A repetição de "não-palavras" (pseudo-palavras) foi utilizada como uma medida mais sensível das habilidades lingüísticas. Observou-se que as crianças com gagueira apresentaram um número maior de erros de fonemas em todas as extensões de "não-palavras", quando comparadas com as crianças sem gagueira. É importante ressaltar que diferenças estatísticas foram registradas somente nas pseudo-palavras de três sílabas. $\mathrm{O}$ desempenho neste teste é consistentemente relacionado a testes convencionais de memória de trabalho fonológica. Concluiu-se que as crianças com gagueira podem ter déficits na habilidade de recordar e/ou reproduzir novas seqüências fonológicas ${ }^{21}$.

Como praticamente todos os outros transtornos de elaboração da linguagem, a gagueira infantil tem um predomínio no sexo masculino. Em crianças com gagueira com menos de cinco anos, a relação entre meninos e meninas é de 2:1 e com o aumento de idade da criança tal relação pode chegar a 3.5 / 1 (a proporção pode diferir de acordo com a faixa etária considerada). As disfluências comuns ocorrem também com maior freqüência nos meninos, embora estas não constituam propriamente um distúrbio ${ }^{8}$. 0 déficit de linguagem provavelmente é um elemento comum entre a gagueira e todos os distúrbios da linguagem $^{5,8,9,12,17,22}$.

Alguns autores observaram que a complexidade fonêmica de alguns idiomas, como a do idioma alemão pode favorecer o desenvolvimento de gagueira assim como influenciar a ocorrência de rupturas ga- gas em palavras de classes gramaticais diversas. $O$ que reforça a concepção de que na gagueira o tempo de ativação e de escolha fonêmica para a fala está prejudicado ${ }^{16,23,24}$.

Observa-se nas crianças com gagueira, mas não nas com disfluências comuns, dificuldades fonoarticulatórias, com contatos articulatórios intensos nos momentos de fala e com variações bruscas na voz, tanto na intensidade quanto na tonalidade. Existe uma hipótese, ainda não comprovada, de que crianças que gaguejam possam ter órgãos fonoarticulatórios menos desenvolvidos. O que se sabe por enquanto é que a fonte primária da gagueira não está no aparelho fonador, apenas causa uma repercussão neste $2,5,7,9,12,17,25,26$.

Em estudos realizados com crianças fluentes préescolares, com e sem antecedentes genéticos para o desenvolvimento da gagueira, observou-se que a herança genética de alguma forma predispõe às rupturas gagas, sem que haja uma relação direta entre a genética e as repercussões no aparelho fonoarticulatório ${ }^{11,27}$.

Estudos revelam também que pode existir associação entre a gagueira e alterações auditivas centrais, mais especificamente com déficits no processamento auditivo central ${ }^{12,18,28,29}$.

\section{Análise Comparativa Quantitativa}

A gagueira infantil distingue-se da disfluência comum na infância também quanto a aspectos quantitativos. As crianças com disfluência comum não manifestam com tanta freqüência rupturas na fala quanto aquelas com gagueira ${ }^{4}$.

Há uma variação dos parâmetros quantitativos entre as idades e entre os sexos feminino e masculino ${ }^{30,31}$. Logo, na avaliação quantitativa, é importante verificar se os dados obtidos nas amostras de fala da criança estão ou não dentro do intervalo de confiança esperado para sua idade e sexo. Para isso é útil consultar Tabelas de Referência para o Perfil da Fluência na faixa etária da criança que está sendo avaliada ${ }^{31}$.

Assim, na faixa etária de 3;0-3;11 (anos; meses), na qual se observa muitas vezes o início de gagueira desenvolvimental, os valores de referência (intervalo de confiança de $95 \%$ ) para o sexo feminino são $0,4-$ 4,1 de disfluências gagas e 12,0-22,4 de disfluências comuns. Para a mesma faixa etária, sexo masculino, esses valores são 1,4-7,4 de disfluências gagas e 12,8-30,1 de disfluências comuns (em uma amostra de 200 sílabas expressas ou fluentes). A variação dos valores de referência, de um modo geral, para as outras idades não é estatisticamente significante quando comparados aos desta faixa etária ${ }^{31}$.

Na gagueira em pré-escolares, observa-se que o total de disfluências gagas e também em geral o total de disfluências comuns ultrapassam os valores 
de referência para idade e sexo, enquanto no quadro de disfluência comum, somente o total de disfluências comuns encontra-se elevado ${ }^{30}$.

A freqüência de rupturas é também um parâmetro quantitativo significativo e inclui a percentagem de descontinuidade de fala e a percentagem de disfluências gagas. Ambas encontram-se acima dos valores de referência nas crianças com gagueira ${ }^{30,31}$.

Dentre os tipos específicos de disfluências, a percentagem de sílabas gaguejadas revela-se como um parâmetro quantitativo de especial relevância e está vinculado com a severidade de gagueira. O percentual acima de 3\% de sílabas gaguejadas é indicativo de gagueira infantil. Sendo que a freqüência de disfluência de sílaba em crianças com gagueira em geral alcança valores mais altos, em torno de 7 a $14 \%$. As estimativas quantitativas devem ser consideradas apenas quando as disfluências gagas puderem ser devidamente documentadas ${ }^{4,5,14,32}$.

Outros parâmetros quantitativos importantes são o fluxo de sílabas por minuto e o fluxo de palavras por minuto. Importantes porque possibilitam saber se a taxa de elocução verbal da criança está ou não próxima do esperado. O fluxo de sílabas por minuto encontra-se em geral bastante reduzido em pré-escolares com gagueira. $\mathrm{O}$ mesmo acontece com o fluxo de palavras por minuto ${ }^{5,30,31}$.

\section{DISCUSSÃO}

O diagnóstico diferencial precoce entre gagueira e disfluência comum na infância tem sido às vezes difícil em decorrência de alguns fatores como:

- Ocorrência de períodos de remissão (às vezes longos) nos estágios iniciais da gagueira infantil, quando o período de intermitência é maior: as características clínicas persistem, porém de forma latente, para somente depois se tornarem novamente evidentes ${ }^{3}$.

- Coincidência entre a faixa etária em que se observa uma maior incidência de início de gagueira e a época do "apogeu" da fala disfluente em crianças no período de aquisição da linguagem ${ }^{8}$.

No entanto, a avaliação fonoaudiológica cuidadosa possibilita detectar diferenças importantes nas crianças com gagueira quando comparadas com as crianças com disfluência comum ${ }^{4-6}$. O que é relevante, já que cerca de metade dos pré-escolares sem gagueira passam por uma fase de acentuação das disfluências comuns ${ }^{10}$.

$\mathrm{Na}$ gagueira, a criança pode ter dificuldade ao acessar um lema (palavra sintática) ou o seu lexema (aspectos fonológicos da palavra), o que poderia causar ou favorecer atrasos importantes e rupturas na estruturação do plano fonético ${ }^{20}$. Assim, muitas disfluências gagas poderiam estar relacionadas com uma instabilidade no planejamento de fones, ou seja, com dificuldades e falhas nos processos envolvidos com a codificação fonológica e ainda, com a formulação lexical ${ }^{8,20,24}$. Observou-se nas crianças com gagueira déficits importantes no preparo das sílabas. Por estas razões, os tipos de disfluência mais encontrados na gagueira infantil são as repetições fônicas e de sílabas (em geral, três vezes ou mais, numa mesma palavra). E também, os bloqueios e os prolongamentos fônicos. Quando ocorrem repetições de palavras inteiras, estas são monossilábicas, na grande maioria das vezes ${ }^{1-5,8,10,12,14-16,20,21,24,33,34}$.

A disfluência comum na infância, por outro lado, decorre principalmente das incertezas passageiras no planejamento lingüístico de palavras pouco familiares e de frases mais complexas, no decorrer dos anos de desenvolvimento da linguagem. Deste modo, pode-se dizer que as disfluências comuns exercem, sobretudo, a função de um momento para que a criança consiga preparar o que pretende dizer ${ }^{8}$, uma vez que ainda não tem precisão na construção sintagmática. As disfluências comuns podem ser também resultado de situações de estresse comunicativo, de ambigüidade na mensagem formulada, do desejo de enfatizar algo na fala, dentre outros fatores. Por isso, as disfluências comuns que ocorrem com maior freqüência são as hesitações e repetições de palavras inteiras e de sintagmas ${ }^{1,2,4-10,14,15,35}$

É importante salientar que a gagueira faz parte de um contexto maior que é o dos distúrbios da comunicação e que os fatores lingüísticos provavelmente estão envolvidos desde o seu início. É possível que dificuldades na construção morfossintática oral e em habilidades semânticas estejam associadas nas crianças pequenas que gaguejam. Pode ser que, para tentar superar as dificuldades sintáticas, elas utilizem na fala somente as palavras cujos conceitos estão melhores estabelecidos para elas. E, com isso, seu campo semântico pareça ser menos extenso do que é na realidade ${ }^{18-20,36}$.

Parece haver consenso na literatura pesquisada de que o preparo da produção fonológica é mais difícil para as crianças que gaguejam. Estas possivelmente têm dificuldades com a memória de trabalho fonológica. E, segundo os autores que forneceram o apoio de pesquisa para esta conclusão, futuros estudos que investiguem tal descoberta podem vir a esclarecer melhor o aparecimento e as características da gagueira na infância ${ }^{1,21,24}$.

Não há evidências de alterações orgânicas no aparelho fonoarticulatório que justifiquem a gagueira em pré-escolares. As dificuldades lingüísticas surgem primeiro e provavelmente como conseqüência destas uma desorganização da motricidade acaba acontecendo. O que agrava a gagueira, levando a uma maior ocorrência de bloqueios e prolongamentos de sons. Nestes tipos de disfluências gaguejadas, os erros fonêmicos parecem estar mais vinculados com a execução motora da fala ${ }^{8,9,17}$. 
Apesar de distúrbio do processamento auditivo central (DPAC) ter sido detectado em crianças com gagueira, não existem evidências de uma correspondência entre o grau de gagueira e o de disfunção auditiva. Estudos mais aprofundados nesta área devem ser realizados ${ }^{18}$.

Uma relação foi verificada entre variáveis fonológicas e o desempenho de pré-escolares, com e sem gagueira, em nomeação de figuras. Todas as crianças manifestaram maior imprecisão e lentidão em palavras fonologicamente densas (aquelas que diferem de outras apenas por um fonema). Pode ser que crianças com DPAC apresentem maior inabilidade na evocação deste tipo de palavras e que isso contribua para o estabelecimento da gagueira, caso haja predisposição genética para tal ${ }^{18,37}$.

A gagueira é um distúrbio quantificável. É importante enfatizar, todavia, que a avaliação quantitativa nem sempre contribui de forma clara para a diferenciação entre a fala disfluente e a fala gaguejada principalmente devido à natureza episódica da gagueira no seu início, em crianças pré-escolares. Logo, este tipo de avaliação deve sempre ser feita em diferentes ocasiões e em associação com a análise dos aspectos qualitativos. Em alguns casos o acompanhamento longitudinal é necessário para o diagnóstico correto. E é fundamental avaliar a fala espontânea da criança em locais e situações em que ela se sinta descontraída já que a obtenção de dados de avaliação no local de atendimento clínico não substitui os dados registrados em condições mais naturais ${ }^{2,3,5,6,10,36}$.

\section{CONCLUSÃO}

Pela revisão de literatura realizada, foi possível concluir que as manifestações clínicas no início do desenvolvimento de gagueira em crianças pequenas (entre dois e seis anos de idade) se assemelham muitas vezes às manifestações próprias da chamada disfluência comum. Contudo, como mostram as pesquisas realizadas com análise qualitativa e/ou quantitativa dos dados clínicos observados nas duas condições, existem diferenças não só na fala como também na linguagem que parecem contribuir para um diagnóstico diferencial preciso. Assim como para a conduta mais adequada para cada caso.

\begin{abstract}
Purpose: to examine the clinical manifestations of stuttering and common dysfluency in their qualitative and quantitative aspects, in children between the ages of two and six years old. Methods: systematic literature review from books and indexed scientific articles on speech pathology in LILACS and MEDLINE from 1993 to 2005. Results: the most important qualitative differences are: the kind of linguistic unit where dysfluency occur, dysfluency typologies, the presence or absence of physical effort during the speech and possible language difficulties. Preschoolers who stutter very often show difficulties in metalinguistic competencies, especially with the metaphonological ones and more studies about this aspect are needed. The frequency of diffluent syllables and the rate of verbal elocution are among the significant quantitative aspects. Conclusion: differences in the speech and in the language seem to be important factors to distinguish stuttering from dysfluency in the childhood.
\end{abstract}

KEYWORDS: Stuttering; Child, Preschool; Child Language; Phonetics; Genetics

\section{REFERÊNCIAS}

1.Barbosa LMG. Noções básicas sobre a gagueira: suas características, sua etiologia e as teorias sobre sua natureza. In: Ribeiro IM, Marchesan IQ, Zorzi JL, organizadores. Conhecimentos essenciais para atender bem a pessoa com gagueira. São José dos Campos: Pulso; 2003. p. 17-32.

2.Bohnen AJ. Avaliando crianças com gagueira. In: Ribeiro IM, Marchesan IQ, Zorzi JL, organizadores. Conhecimentos essenciais para atender bem a pessoa com gagueira. São José dos Campos: Pulso; 2003. p. 41-54.
3.Barbosa LMG, Chiari BM. Gagueira: etiologia, prevenção e tratamento. 2. ed. Barueri: Pró-Fono; 2005. 4.Boone D, Plante E. Comunicação humana e seus distúrbios. 2 ed. Porto Alegre: Artes Médicas; 1994. 5.Andrade CRF. Diagnóstico e intervenção precoce no tratamento das gagueiras infantis. Barueri: PróFono; 2004.

6. Andrade CRF. Protocolo para avaliação da fluência da fala. São Paulo: Pró-Fono; 2004.

7. Jakubovicz R. Agagueira: teoria e tratamento de adultos e crianças. 5. ed. Rio de Janeiro: Revinter; 1997. 8.Degiovani VM, Chiari BM, Schiefer AM. Disfluência: caracterização dos tipos e freqüência de ocorrência em 
um grupo de escolares. Pró-Fono. 1999; 11(1):32-7. 9. Launay CL, Maisonny SB. Distúrbios da linguagem, da fala e da voz na infância. São Paulo: Roca; 1989.

10. Sassi FC, Campanatti-Ostiz H, Andrade CRF. Terminologia: fluência e desordens da fluência. PróFono. 2001; 13(1):107-13.

11. Andrade CRF. História natural da gagueira: estudo I: perfil da fluência. Pró-Fono. 2002; 14(3):351-60. 12. Andrade CRF. Gagueiras infantis: atualização sobre a determinação de fatores de risco e condutas. Pediatria. 1997; 19(2):150-8.

13. Weir E, Bianchet S. Developmental dysfluency: early intervention is key. CMAJ. 2004; 170(12):1790-1. 14. Zackiewicz D, Andrade CRF. Seis parâmetros da fluência. Rev Soc Bras Fonoaudiol. 2000; 7:59-64.

15. Van Riper C, Emerick L. Correção da linguagem: uma introdução à patologia da fala e à audiologia. 8 . ed. Porto Alegre: Artes Médicas; 1997.

16. Natke U, Sandrieser P, Van Ark M, Pietrowsky $\mathrm{R}$, Kalveram KT. Linguistic stress, within-word position, and grammatical class in relation to early childhood stuttering. J Fluency Disord. 2004; 29(2):109-22.

17. Dinville C. A gagueira: sintomatologia e tratamento. Rio de Janeiro: Enelivros; 1993.

18. Schiefer AM, Barbosa LMG, Pereira LD. Considerações preliminares sobre uma possível correlação entre a gagueira e os aspectos lingüísticos e auditivos. Pró-Fono. 1999; 11(1):27-31.

19. BajajA, Hodson B, Schommer-Aikins M. Performance on phonological and grammatical awareness metalinguistic tasks by children who stutter and their fluent peers. J Fluency Disord. 2004; 29(1):63-77.

20. Anderson JD, Conture EG. Sentence-structure priming in young children who do and do not stutter. J Speech Lang Hear Res. 2004; 47(3):552-71.

21. Hakim HB, Ratner NB. Nonword repetition abilities of children who stutter: an exploratory study. J Fluency Disord. 2004; 29(3):179-99.

\section{RECEBIDO EM: 13/10/2006 \\ ACEITO EM: 07/05/2007}

Endereço para correspondência:

Rua Gal Pereira da Silva, 25 -Apto. 1101 M

Niterói - RJ

CEP: $24220-030$

Tel: (21) 27042749

E-mail:mercon@uol.com.br
22. Nippold MA. Phonological and language disorders in children who stutter: impact on treatment recommendations. Clin Linguist Phon. 2004; 18(2):145-59.

23. Dworzynski K, Howell P. Predicting stuttering from phonetic complexity in German. J Fluency Disord. 2004; 29(2):149-73.

24. Andrade CRF. História natural da gagueira: estudo III: vocabulário, fonologia e pragmática. Pró-Fono. 2002; 14(3):371-82.

25. Melnick KS, Conture EG, Ohde RN. Phonological priming in picture naming of young children who stutter. J Speech Lang Hear Res. 2003; 46(6):1428-43.

26. Goldfeld M. Fundamentos em fonoaudiologia: linguagem. 2. ed. Rio de Janeiro: Guanabara Koogan; 2003.

27. Andrade CRF. História natural da gagueira: estudo Il: sistema miofuncional oral e funções. Pró-Fono. 2002; 14(3):361-70.

28. Schiefer AM. Gagueira: caracterização das disfluências e possíveis correlações com a audição e outros aspectos da fala [doutorado]. São Paulo (SP): Universidade Federal de São Paulo; 1999.

29. Andrade CRF, Schochat E. Comparação entre os achados neurolinguísticos e neuroaudiológicos nas gagueiras. Pró-Fono. 1999; 11(2):27-30.

30. Andrade CRF, Juste F. Proposta de análise de performance e de evolução em crianças com gagueira desenvolvimental. Rev CEFAC. 2005; 7(2):158-70.

31. Andrade CRF. Fluência (parte C). In: Andrade CRF, Béfi-Lopes DM, Fernandes FDM, Wertzner HF. $A B F W$ : teste de linguagem infantil nas áreas de fonologia, vocabulário, fluência e pragmática. 2. ed. Barueri: Pró-Fono; 2004. p. 51-82.

32. Riley J, Riley G, Maguire G. Subjective screening of stuttering severity, locus of control and avoidance: research edition. J Fluency Disord. 2004; 29(1):51-62. 33. Andrade CRF. A história natural da gagueira: estudo dos 0 aos 24 meses de vida. Pró-Fono. 2000; 12(1):60-8.

34. Venkatagiri HS. Slower and incomplete retrieval of speech motor plans is the proximal source of stuttering: stutter occur when syllable motor plans stored in memory are concatenated to produce the utterance motor plan. Med Hypotheses. 2004; 62(3):401-5.

35. Perelló J. Transtornos da fala. Rio de Janeiro: Médsi; 1995.

36. Weiss $A L$. What child language research may contribute to the understanding and treatment of stuttering. Lang Speech Hear Serv Sch. 2004; 35(1):90-2.

37. Arnold HS, Conture EG, Ohde RN. Phonological neighborhood density in the picture naming of young children who stutter: preliminary study. J Fluency Disord. 2005; 30(2):125-48. 